\title{
Generalization of responses to intermediate size by cats and monkeys
}

\author{
J. M. WARREN AND HARVEY C. EBEL ${ }^{2}$ \\ THE PENNSYL VANIA STATE UNIVERSITY
}

After learning to choose the middle sized of three squares, 16 cats and 14 rhesus monkeys manifested much less generalization of response on 10 equivalence tests than 16 control cats trained to select the smallest or the largest of three stimuli. These results are more compatible with Spence's theory of transposition than with relational theories of transposition.

The purpose of this experiment was to investigate the generalization of response to intermediate size by cats and monkeys both on transposition tests and on equivalence tests with objects differing in shape or brightness from those used in initial training, in order to assess the relative importance of relational and absolute cues in the solution of the intermediate size problem.

\section{METHOD}

The 32 cats and 14 rhesus monkeys studied were experienced in visual discrimination learning, but none had previously served in any experiment on transposition or equivalence. All of the testing was carried out in the WGTA. Four vertically oriented black squares with areas of $25,50,100$, and $200 \mathrm{~cm}^{2}$ were used in discrimination training and transposition testing. The objects used in the equivalence tests are illustrated in Fig. 1; each of the shapes used in Series 1 through 6 was equated with the corresponding member of the training series in linear extent. This was not true for the variable figures (F) or grounds (G) of the stimuli in Series 7 and 8, which thus combined features of traditional transposition and equivalence tests. The white squares of Series 1 were presented on a black test tray; a white test tray was used in all other phases of the experiment.

Procedure-Intermediate Size Groups

Cats often fall to learn the intermediate size problem in a tolerable number of trials if they are tested on three-choice configurations from the outset of training (Warren, 1965). Elght of the 16 cats trained on intermediate size were, therefore, taught the problem in two stages.

Pretraining. Four cats were trained with stimulus Set 1 (25-50-100 $\left.\mathrm{cm}^{2}\right)$ and four with set $2(50-100-$ $200 \mathrm{~cm}^{2}$ ). In pretraining, each group was trained to choose the intermediate sized square when it was paired with either the largest or smallest stimulus in two-choice presentations. The Ss were tested 45 noncorrection trials a day to a criterion of 15 consecutive correct responses within a single session.

Intermediate Size Training. The eight pretrained cats were then required to discriminate the same set of stimuli when all three were presented simultaneously on every trial. All of the monkeys and elght cats were trained on this latter task from the outset, half with Set 1 and half with Set 2, and received 45 noncorrection trials per day to a criterion of 15 consecutive correct responses in a single session.

Three cats which failed to learn the three-cholce intermediate size problem in 1800 trials were switched to the two-choice (Pretraining) task, trained to criterion and then retested on the three-choice problem until the criterion was attained.

Transposition Tests. The monkeys and cats trained on

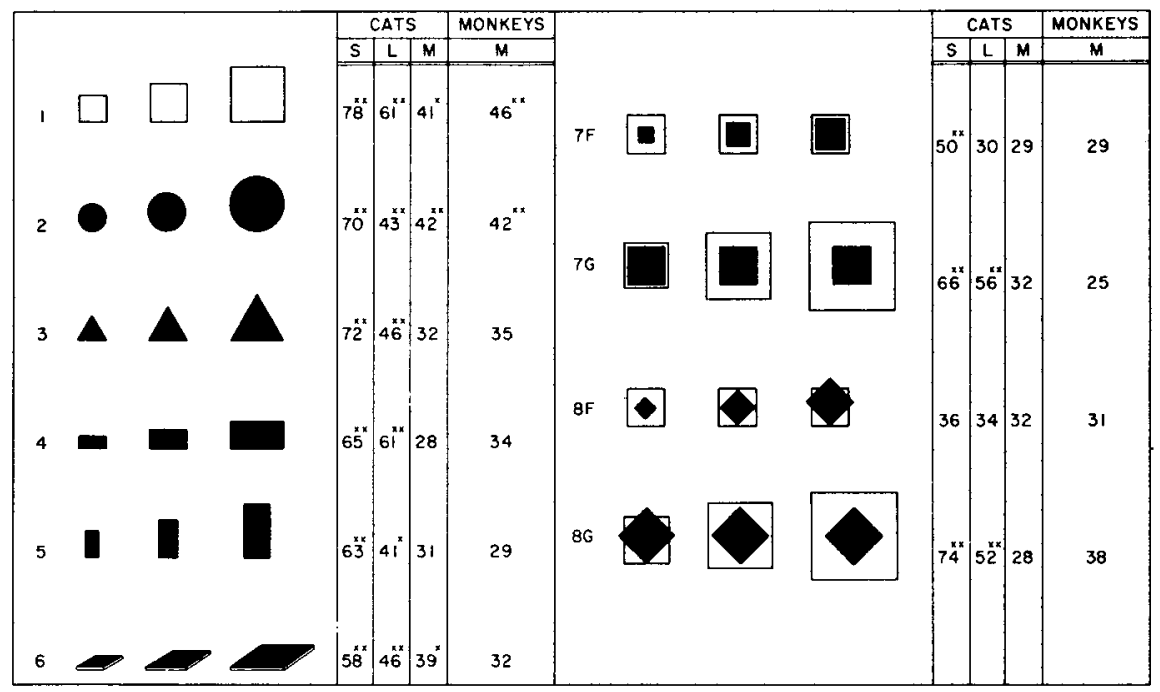

Fig. 1. Percentage of equivalence responses by Ss trained to choose the smallest (S), largest (L) or middle-sized (M) of three squares; $X$ and $X X$ indicate $p$ 's of .01 and .001 , respectively. 
intermediate size were next tested on a series of six transposition tests previously described by Warren (1965). The cats were given 45 and the monkeys 15 trials in each of these tests.

Equivalence Tests. After completion of the Transposition Tests, the Ss were retrained to criterion with the set of stimuli on which they had been trained prior to the last transposition test. They were then tested for equivalence responses with the eight sets of test stimuli shown in Fig. 1; the order in which the tests were given was counterbalanced across Ss by means of latin squares. Each equivalence test session consisted of 40 trials; the training stimuli were presented and differentially reinforced on Trials 1-10 and 21-30 to maintain the discrimination, and the test figures were presented on Trials 11-20 and 31-40 with all three stimuli reinforced. Double pairs of test stimuli were used in Equivalence Tests 7 and 8. Each $F$ (figure variable) and $G$ (ground variable) set of stimuli was presented in the first or second series of 10 test trials for half the Ss.

\section{Procedure-Confrol Group}

Early observations on animals trained on intermediate size indicated a relative failure of transfer on the equivalence tests. It seemed therefore worthwhile to exclude the possibility that the equivalence figures were not discriminably different by testing animals trained to choose the largest or smallest rather than the middle sized of three objects. Eight cats were trained with Set 1 and eight with Set 2, half of the Ss being required to select the smallest, and half the largest of the three squares. All were trained 45 trials per day to a criterion of 15 consecutive correct responses and then tested on the equivalence tests.

\section{RESULTS}

\section{Training}

Median total trials to criterion for the major groups of Ss were as follows: cats trained on intermediate size, 1670; monkeys trained on intermediate size, 135; and cats trained to choose the largest or smallest stimulus, 355. These findings confirm previous observations that intermediate size is a very difficult problem for cats (Warren, 1965).

\section{Transposifion}

Both cats and monkeys trained on intermediate size responded predominantly to absolute rather than relative cues on all transposition tests. The mean percentage of relative and absolute choices by the cats was 20 and 76; the corresponding values for the monkeys were 23 and 70, respectively. These findings replicate those obtained in previous studies with chimpanzees (Spence, 1942) and cats (Warren, 1965).

\section{Equivalence}

The results of the equivalence tests are summarized in Fig. 1 which shows for each group the percentage of appropriate equivalence responses to each set of test stimuli and which percentages differ significantly from chance, as determined by the binomial test. The cats that learned to choose the middle sized stimulus
(M) showed significant transfer on only three sets of potentially equivalent figures, and the monkeys on only two. The cats trained to choose the largest or smallest stimulus, in contrast, showed significant transfer on all sets of objects differing in external contour. Overall, the mean percentages of equivalence responses by the cats trained to the middle size, largest, and smallest stimuli were 34,48 , and 64 , respectively. The Jonckheere (1954) test indicates the ordered differences among groups is significant with $p=.001$ $(z=5.99)$. The more successful transfer by cats trained on the smallest stimulus probably reflects the preference for small size shown in previous experiments with carnivores (Ebel \& Werboff, 1967; Warren \& Sinha, 1957).

\section{DISCUSSION}

The major new finding of this experiment is that neither cats nor monkeys trained to choose the middle sized of three squares show significant transfer, when tested with shapes other than squares, to the same extent as cats trained to select the largest or smallest of three squares.

Zeiler (1965) found that children trained to choose the intermediate of three stimuli differing in size learn more slowly than Ss required to choose the largest or smallest, and that the children trained to select the middle size made many fewer relative transposition responses than those trained with the largest or smallest correct. His findings and those of the present study agree in indicating that learning to select the intermediate size is a distinctly different task for $S$ than is learning to choose the largest or smallest of three stimuli. This conclusion seems difficult to reconcile with theories that seek to explain transposition in cognitive or relational terms, but it seems qualitatively quite compatible with Spence's (1942) view that the solution of the intermediate size problem requires very precise differentiation of absolute stimulus values.

\section{References}

EBEL, H. C., \& WERBOFF, J. Transposition in dogs: Successive reversals of the intermediate size problem. Percept. mot. Skills, 1967, 24, 507-511.

JONCKHEERE, A. R. A distribution-free K-sample test against ordered alternatives. Biometrika, 1954, 41, 133-145.

SPENCE, K. W. The basis of solution by chimpanzees of the intermediate size problem. J. exp. Psychol., 1942, 31, 247-271.

WARREN, J. M. Primate learning in comparative perspective. In A. M. Schrier, H. F. Harlow, \& F. Stollnitz (Eds.), Behavior of nonhuman primates: Modern research trends. Vol. 1. New York: Academic Press, 1965. Pp. 249-281.

WARREN, J. M., \& SINHA, M. M. Effect of differential reinforcement on size preferences in cats. Percept. mot. Skills, 1957, 7, 17-22.

ZEILER, M. Transposition after training to the largest, smallest or middle-sized of three stimuli. Paper presented at Eastern Psychological Association meetings, 1965.

\section{Notes}

1. This research was supported by Grant MH-04726 from the National Institute of Mental Health, U. S. Public Health Service.

2. Now at Laboratory of Neurophysiology, Columbia University College of Physicians and Surgeons. 